\title{
Hypertension Is Related to Positive Global Sagittal Alignment: A Cross-Sectional Cohort Study
}

\author{
Hideyuki Arima ${ }^{1}$, Daisuke Togawa ${ }^{2}$, Tomohiko Hasegawa ${ }^{1}$, Yu Yamato $^{1}$, Go Yoshida ${ }^{1}$, Sho Kobayashi ${ }^{1}$, \\ Tatsuya Yasuda ${ }^{3}$, Tomohiro Banno ${ }^{1}$, Shin $\mathrm{Oe}^{2}$, Yuki Mihara ${ }^{1}$, Hiroki Ushirozako ${ }^{1}$, Hironobu Hoshino ${ }^{1}$, \\ Yukihiro Matsuyama ${ }^{1}$
}

\begin{abstract}
${ }^{I}$ Department of Orthopedic Surgery, Hamamatsu University School of Medicine, Hamamatsu, Japan ${ }^{2}$ Division of Geriatric Musculoskeletal Health, Hamamatsu University School of Medicine, Hamamatsu, Japan

${ }^{3}$ Department of Orthopedic Surgery, Hamamatsu Medical Center, Hamamatsu, Japan
\end{abstract}

\section{Study Design: Cross-sectional cohort study.}

Purpose: This study aimed to investigate the relationship between hypertension and spino-pelvic sagittal alignment in middle-aged and elderly individuals.

Overview of Literature: Positive global sagittal alignment is associated with poor health-related quality of life. Hypertension is associated with tissue microcirculation disorders of the skeletal muscle. We hypothesized that hypertension may be involved in positive global sagittal alignment.

Methods: In this institutional review board-approved study, 655 participants $(262$ men and 393 women; mean age, 72.9 years; range, 50-92 years) who underwent musculoskeletal screening in Toei town, Aichi, Japan were included. Whole spine and pelvic radiographs were taken, and radiographic parameters (thoracic kyphosis, lumbar lordosis, pelvic tilt, sacral slope, pelvic incidence, and sagittal vertical axis [SVA]) were measured using an image-analysis software. Hypertension was assessed using the standard criteria. The study participants were divided into three subgroups as per age (50-64 years, $65-74$ years, and $\geq 75$ years). We examined the differences in the radiographic parameters of those with and without hypertension in each age subgroup.

Results: In each age subgroup, there was no significant difference in the age and sex of those with and without hypertension. SVA was significantly shifted forward in the hypertension group than in the non-hypertension group in those aged 50-64 years old (32.4 $\mathrm{mm}$ vs. $16.0 \mathrm{~mm}, p=0.018$ ) and in those aged $65-74$ years old ( $42.7 \mathrm{~mm}$ vs. $30.6 \mathrm{~mm}, p=0.012$ ). There was no significant difference between the hypertension and non-hypertension groups in terms of the alignment of the lumbar and thoracic spine in all the subgroups. In multivariate analysis, hypertension was a significant independent factor of forward-shifted SVA (standardized beta $0.093, p=0.015$ ). Conclusions: This study showed that hypertension was associated with forward-shifted global sagittal alignment.

Keywords: Adult spinal deformity; Spino-pelvic parameters; Elderly volunteer; Sagittal vertical axis; Hypertension

\section{Introduction}

In recent years, with the increase in the elderly popula- tion, poor health-related quality of life (QOL) owing to spinal deformities in middle-aged and elderly people is becoming a problem $[1,2]$. Changes in the posture are re-

Received Dec 1, 2018; Revised Mar 3, 2019; Accepted Mar 7, 2019

Corresponding author: Hideyuki Arima

Department of Orthopedic Surgery, Hamamatsu University School of Medicine, 1-20-1, Handayama, Higashi-ku, Hamamatsu-city, Shizuoka, 431-3192 Japan

Tel: +81-53-435-2299, Fax: +81-53-435-2296, E-mail: arihidee@yahoo.co.jp 
ported to start in the 30s in women and the 50s in men [3]. In addition, previous cohort studies have reported that the proportion of people who exceed the threshold value of sagittal global alignment increases in the 60s [4]. If global sagittal alignment shifts forward, it causes deterioration of the health-related QOL $[1,5]$. Deterioration of the healthrelated QOL in middle-aged and elderly people is a very important issue because it leads to increased resource consumption for medical and long-term care. The cause of the forward shift in the sagittal global alignment is considered to be disc degeneration and osteoporotic vertebral body fracture, except for the iatrogenic flat back syndrome $[6,7]$. Although it was reported that disc degeneration and/or herniation are associated with spinal sagittal alignment $[8,9]$, the root cause is yet to be identified [8]. Risk factors for disc degeneration include high blood pressure [10], impaired glucose tolerance [10,11], hyperlipidemia $[10,11]$, and obesity [10]. These diseases are associated with arteriosclerosis [12], and not only increase the risk of cardiovascular, but also impact the musculoskeletal system due to lack of tissue microcirculation [13]. These factors may directly affect the global sagittal alignment, resulting in intervertebral disc degeneration. In fact, the impact of arteriosclerotic factors on the intervertebral disc is larger in the thoracic vertebrae that are less movable than the lumbar vertebrae [10]. Differences in the impact of arteriosclerotic factors on disc degeneration based on the site suggest that arteriosclerosis factors are indirectly related to disc degeneration. It is unclear whether these arteriosclerosis factors are actually involved in the alteration of spinal alignment; therefore, it is important to elucidate the relationship of these arteriosclerosis factors with sagittal plane alignment. We focused on hypertension, the most common arteriosclerotic factors. This study aimed to clarify whether there is a difference in the thoracic, lumbar, and global sagittal alignment in middle-aged and elderly individuals with and without hypertension.

\section{Materials and Methods}

\section{Participants}

This study was reviewed and approved by the Hamamatsu University School of Medicine institutional review board (IRB approval no., 15-60) and the Toei Hospital IRB (no., 201201). After obtaining approval for the research from the IRB, musculoskeletal examination and radiographic analyses were performed for people in Toei town, Aichi prefecture, Japan in 2012. Toei town has a population of 3,528 (regional official data from October 2011) and is located in the mountains. The present study, entitled the “TOEI study 2012", was conducted as part of a musculoskeletal screening project for middle-aged and elderly individuals in Toei town. Participants aged $>50$ years were enrolled in this study. We obtained written informed consent from the participants for publishing their data. We investigated the following factors: age, sex, smoking status, physical exam (height, weight, body weight index, and blood pressure), radiographic examination, presence of low-back pain, health-related QOL (using the [Oswestry Disability Index [ODI] and the EuroQol 5 dimension [EQ-5D]), and history of cerebrovascular disease and heart disease $[14,15]$.

\section{Hypertension}

Blood pressure was measured twice by experienced nurses, and the average value was calculated. Using the criteria of the Japanese Society of Hypertension, hypertension was defined as a systolic blood pressure of $\geq 140 \mathrm{~mm} \mathrm{Hg}$ or a diastolic blood pressure of $\geq 90 \mathrm{~mm} \mathrm{Hg}$. We also defined the participants who regularly took antihypertensive medication as hypertensive.

\section{Radiographic measurements}

In order to evaluate spinal and pelvic alignment, standing upright full-length posteroanterior and lateral spine radiographs were taken. Radiographic films were obtained with a $1.5-\mathrm{m}$ distance between the $\mathrm{X}$-ray tube and the radiograph for all the participants. The standing posture was standardized; participants were asked to relax their heads while looking straight ahead without pulling in the chin and place their hands on the clavicles. The spino-pelvic parameters (thoracic kyphosis [TK, T5-T12], lumbar lordosis [LL, L1-S1], pelvic tilt [PT], sacral slope, pelvic incidence $[\mathrm{PI}]$, and sagittal vertical axis [SVA]) were measured twice using standard techniques [16]. The intra-and inter-observer reliability was examined using the intraclass correlation coefficient (ICC) in SVA, PT, and PI in this cohort previously. Moreover, the intra-observer ICCs for SVA, PT, and PI were 0.995, 0.996, and 0.918, respectively, and the inter-observer ICCs were 0.996, 0.990, and 0.966 , respectively [17]. We excluded participants who 
had undergone previous spine or joint surgeries (total hip/knee replacement or femoral neck fracture surgery). Radiographic parameters that were measured with poor quality and were very difficult to assess accurately were excluded from the study. Anatomical variations with four or six lumbar vertebrae were also excluded because these factors are significantly related with the variations in the radiographic parameters.

\section{Data analysis}

We divided all the participants into three age subgroups (non-elderly, 50-64 years old; young-old, 65-74 years old; old-old, $\geq 75$ years old) and examined the differences in the radiographic parameters of the hypertension group and non-hypertension group for each age group. Similarly, differences in the average age, physical parameters (height, weight, and body mass index [BMI]), and health-related QOL (ODI and EQ-5D) were also examined between the hypertension and non-hypertension groups for each age subgroup.

\section{Statistical analysis}

All the values are expressed as mean \pm standard deviation. The normal distribution of the data was evaluated using the Shapiro-Wilk test. Differences between the groups were evaluated using the unpaired two-sample $t$-test or Mann-Whitney test. Fisher exact test and chi-square were used to test for significant differences in the categorical study parameters between both the groups. Spearman correlations were used to evaluate the associations of SVA with age, height, and BMI. Multiple regression analysis was used to determine the independent predictors of SVA, TK, LL, and PT with age, sex, height, BMI, presence of hypertension, presence of low-back pain, and history of cerebrovascular disease and heart disease as independent variables. In the multiple regression analysis, multicollinearity was assessed as negative based on a variance inflation factor $<10$. A $p$-value $<0.05$ was considered statistically significant. Statistical analyses were performed using IBM SPSS statistics software ver. 24.0 (IBM Corp., Armonk, NY, USA).

\section{Results}

\section{Participant background}

Among 724 participants, 655 (262 men and 393 women; mean age, 72.9 years; range, 50-92 years) were enrolled in this study. Table 1 shows the age, height, weight, BMI, systolic blood pressure, diastolic blood pressure, and smoking history as per sex. The height $(162.0 \mathrm{~cm}$ versus 148.6 $\mathrm{cm}, p<0.001$ ), weight (60.0 kg versus $49.3 \mathrm{~kg}, p<0.001$ ), and BMI $\left(22.8 \mathrm{~kg} / \mathrm{m}^{2}\right.$ versus $\left.22.3 \mathrm{~kg} / \mathrm{m}^{2}, p=0.044\right)$ of men was significantly higher than that of women. In contrast, systolic blood pressure was significantly higher in women (135.5 mm Hg versus $141.6 \mathrm{~mm} \mathrm{Hg}, p<0.001$ ). Smoking history was significantly more common in men $(53.8 \%$ versus $2.6 \%, p<0.001)$. There was no significant sex-based difference in the prevalence of hypertension ( $71.0 \%$ versus $69.5 \%, p=0.676)$ or low-back pain $(57.3 \%$ versus $64.5 \%$, $p=0.060$ ) (Table 1).

Table 1. Background characteristics of the participants

\begin{tabular}{|c|c|c|c|c|}
\hline Characteristic & All $(n=655)$ & Male $(n=262)$ & Female $(\mathrm{n}=393)$ & $p$-value ${ }^{\text {a) }}$ \\
\hline Age (yr) & $72.9 \pm 8.0$ & $73.2 \pm 8.4$ & $72.7 \pm 7.8$ & 0.286 \\
\hline Height (cm) & $154.0 \pm 9.2$ & $162.0 \pm 6.9$ & $148.6 \pm 6.1$ & $<0.001$ \\
\hline Weight (kg) & $53.6 \pm 9.9$ & $60.0 \pm 9.6$ & $49.3 \pm 7.5$ & $<0.001$ \\
\hline Body mass index $\left(\mathrm{kg} / \mathrm{m}^{2}\right)$ & $22.5 \pm 2.9$ & $22.8 \pm 2.8$ & $22.3 \pm 3.0$ & 0.044 \\
\hline Systolic blood pressure (mm Hg) & $139.2 \pm 18.8$ & $135.5 \pm 17.5$ & $141.6 \pm 19.3$ & $<0.001$ \\
\hline Diastolic blood pressure $(\mathrm{mm} \mathrm{Hg})$ & $77.4 \pm 11.9$ & $77.9 \pm 12.8$ & $77.0 \pm 11.1$ & 0.346 \\
\hline Hypertension prevalence (\%) & 70.1 & 71.0 & 69.5 & 0.676 \\
\hline Low-back pain prevalence (\%) & 61.6 & 57.3 & 64.5 & 0.060 \\
\hline Smoking history (\%) & 23.1 & 53.8 & 2.6 & $<0.001$ \\
\hline
\end{tabular}

Values are presented as mean \pm standard deviation, unless otherwise stated. Values in boldface indicate statistical significance.

${ }^{a}$ Comparison between male and female; $p<0.05$ was considered as significant. 
Table 2. Difference in spino-pelvic parameter between hypertension and non-hypertension group among 50 to 64 years old population

\begin{tabular}{|c|c|c|c|}
\hline Variable & Hypertension (n=67) & Non-hypertension $(n=50)$ & $p$-value ${ }^{\text {a) }}$ \\
\hline Age (yr) & $60.6 \pm 4.0$ & $60.7 \pm 3.7$ & 0.869 \\
\hline Female percentage $(\%)$ & 56.7 & 68.0 & 0.215 \\
\hline Height (cm) & $159.2 \pm 9.0$ & $158.0 \pm 8.9$ & 0.315 \\
\hline Weight (kg) & $61.5 \pm 13.7$ & $53.1 \pm 8.3$ & $<0.001$ \\
\hline Body mass index $\left(\mathrm{kg} / \mathrm{m}^{2}\right)$ & $24.1 \pm 3.8$ & $21.2 \pm 2.1$ & $<0.001$ \\
\hline Systolic blood pressure (mm Hg) & $145.6 \pm 16.3$ & $120.4 \pm 13.0$ & $<0.001$ \\
\hline Diastolic blood pressure $(\mathrm{mm} \mathrm{Hg})$ & $86.9 \pm 10.6$ & $74.1 \pm 8.7$ & $<0.001$ \\
\hline Low-back pain prevalence (\%) & 64.2 & 51.0 & 0.155 \\
\hline Smoking history (\%) & 20.9 & 20.0 & 0.906 \\
\hline \multicolumn{4}{|l|}{ Spino-pelvic parameter } \\
\hline Thoracic kyphosis $\left({ }^{\circ}\right)$ & $33.8 \pm 12.8$ & $32.0 \pm 10.2$ & 0.383 \\
\hline Lumbar lordosis $\left(^{\circ}\right)$ & $43.5 \pm 11.5$ & $44.3 \pm 14.5$ & 0.366 \\
\hline Pelvic tilt $\left(^{\circ}\right)$ & $15.1 \pm 8.1$ & $14.0 \pm 6.5$ & 0.673 \\
\hline Sacral slope $\left(^{\circ}\right)$ & $32.8 \pm 8.5$ & $33.1 \pm 10.0$ & 0.506 \\
\hline Pelvic incidence $\left(^{\circ}\right)$ & $47.8 \pm 9.9$ & $46.1 \pm 10.4$ & 0.604 \\
\hline Pelvic incidence minus lumbar lordosis $\left({ }^{\circ}\right)$ & $4.4 \pm 12.9$ & $1.8 \pm 10.1$ & 0.241 \\
\hline Sagittal vertical axis (mm) & $32.4 \pm 34.0$ & $16.0 \pm 23.8$ & 0.018 \\
\hline \multicolumn{4}{|l|}{ Health-related quality of life parameter } \\
\hline Euro0ol 5 dimension & $0.91 \pm 0.13$ & $0.92 \pm 0.12$ & 0.731 \\
\hline Oswestry Disability Index & $8.3 \pm 9.7$ & $6.9 \pm 8.7$ & 0.484 \\
\hline
\end{tabular}

Values are presented as mean \pm standard deviation, unless otherwise stated. Values in boldface indicate statistical significance.

${ }^{a}$ Comparison between hypertension group and non-hypertension group; $p<0.05$ was considered as significant.

\section{Differences in the spino-pelvic parameters of the hy- pertension and non-hypertension groups}

1) Non-elderly group (50-64 years old)

There were no statistically significant differences in the age, sex, height, prevalence of low-back pain, and smoking history of the hypertension and non-hypertension groups. Weight and BMI were significantly higher in the hypertension group. Among the spino-pelvic parameters, there was no significant difference in TK $\left(33.8^{\circ}\right.$ versus $\left.32.0^{\circ}, p=0.383\right)$, LL ( $43.5^{\circ}$ versus $\left.44.3^{\circ}, p=0.366\right)$, and PT $\left(15.1^{\circ}\right.$ versus $\left.14.0^{\circ}, p=0.673\right)$. In contrast, the SVA was significantly shifted forward in the hypertension group (32.4 mm versus $16.0 \mathrm{~mm}, p=0.018$ ) (Table 2 ).

2) Young-old group (65-74 years old)

There were no statistically significant differences in the age, sex, height, prevalence of low-back pain, and smoking history of the hypertension and non-hypertension groups. Weight and BMI were significantly higher in the hypertension group. Among the spino-pelvic parameters, there was no significant difference in TK $\left(34.3^{\circ}\right.$ versus $\left.33.3^{\circ}, p=0.536\right)$, LL ( $41.9^{\circ}$ versus $\left.41.5^{\circ}, p=0.849\right)$, and PT $\left(18.1^{\circ}\right.$ versus $\left.17.3^{\circ}, p=0.401\right)$. However, the SVA was significantly shifted forward in the hypertension group (42.7 $\mathrm{mm}$ versus $30.6 \mathrm{~mm}, p=0.012$ ) (Table 3 ).

\section{3) Old-old group ( $\geq 75$ years)}

There were no statistically significant differences in the age, sex, prevalence of low-back pain, or smoking history of the hypertension and non-hypertension groups. Weight and BMI were significantly higher in the hypertension group. Those in the non-hypertension group were significantly taller. Among the spino-pelvic parameters, there was no significant difference in TK $\left(37.7^{\circ}\right.$ versus $38.3^{\circ}$, $p=0.773)$, LL $\left(37.1^{\circ}\right.$ versus $\left.38.9^{\circ}, p=0.780\right)$, PT $\left(22.3^{\circ}\right.$ versus $\left.20.1^{\circ}, p=0.161\right)$, or SVA $(70.5 \mathrm{~mm}$ versus 57.3 $\mathrm{mm}, p=0.063$ ) (Table 4). 
Table 3. Difference in spino-pelvic parameter between hypertension and non-hypertension group among 65 to 74 years old population

\begin{tabular}{|c|c|c|c|}
\hline Variable & Hypertension ( $n=165)$ & Non-hypertension ( $\mathrm{n}=84$ ) & $p$-value ${ }^{\text {a) }}$ \\
\hline Age (yr) & $70.5 \pm 2.6$ & $70.2 \pm 2.7$ & 0.544 \\
\hline Female percentage (\%) & 60.0 & 66.7 & 0.305 \\
\hline Height (cm) & $155.5 \pm 9.4$ & $154.0 \pm 7.7$ & 0.219 \\
\hline Weight (kg) & $56.2 \pm 9.3$ & $51.6 \pm 8.6$ & $<0.001$ \\
\hline Body mass index $\left(\mathrm{kg} / \mathrm{m}^{2}\right)$ & $23.2 \pm 2.7$ & $21.7 \pm 2.6$ & $<0.001$ \\
\hline Systolic blood pressure (mm Hg) & $144.9 \pm 16.3$ & $124.2 \pm 11.6$ & $<0.001$ \\
\hline Diastolic blood pressure (mm Hg) & $81.3 \pm 11.1$ & $71.3 \pm 8.7$ & $<0.001$ \\
\hline Low-back pain prevalence (\%) & 55.2 & 57.1 & 0.765 \\
\hline Smoking history (\%) & 29.7 & 19.0 & 0.070 \\
\hline \multicolumn{4}{|l|}{ Spino-pelvic parameter } \\
\hline Thoracic kyphosis $\left({ }^{\circ}\right)$ & $34.3 \pm 13.1$ & $33.3 \pm 11.9$ & 0.536 \\
\hline Lumbar lordosis $\left(^{\circ}\right)$ & $41.9 \pm 15.2$ & $41.5 \pm 14.0$ & 0.849 \\
\hline Pelvic tilt $\left({ }^{\circ}\right)$ & $18.1 \pm 8.1$ & $17.3 \pm 8.5$ & 0.401 \\
\hline Sacral slope $\left({ }^{\circ}\right)$ & $32.7 \pm 11.1$ & $31.7 \pm 10.4$ & 0.749 \\
\hline Pelvic incidence $\left({ }^{\circ}\right)$ & $49.5 \pm 11.5$ & $47.9 \pm 12.6$ & 0.346 \\
\hline Pelvic incidence minus lumbar lordosis $\left({ }^{\circ}\right)$ & $7.6 \pm 15.0$ & $6.3 \pm 11.8$ & 0.660 \\
\hline Sagittal vertical axis (mm) & $42.7 \pm 38.6$ & $30.6 \pm 28.6$ & 0.012 \\
\hline \multicolumn{4}{|l|}{ Health-related quality of life parameter } \\
\hline Euro0ol 5 dimension & $0.84 \pm 0.16$ & $0.86 \pm 0.15$ & 0.417 \\
\hline Oswestry Disability Index & $11.2 \pm 11.3$ & $10.5 \pm 10.8$ & 0.957 \\
\hline
\end{tabular}

Values are presented as mean \pm standard deviation, unless otherwise stated. Values in boldface indicate statistical significance.

${ }^{a}$ Comparison between hypertension group and non-hypertension group; $p<0.05$ was considered as significant.

3. Relationship of sagittal vertical axis and lumbar lordosis with age, sex, height, body mass index, and hypertension

Univariate analysis showed that SVA increased with age $(r=0.424, p<0.001)$. There was no difference in the SVA of men and women $(48.3 \pm 44.6 \mathrm{~mm}$ versus $49.6 \pm 47.9$ $\mathrm{mm}, p=0.989)$. Univariate analysis showed that SVA decreased with height $(r=-0.169, p<0.001)$. SVA was not correlated with BMI $(r=0.054, p=0.166)$ and was higher in the hypertension group than in the non-hypertension group (54.9 $\pm 49.2 \mathrm{~mm}$ versus $35.3 \pm 36.1 \mathrm{~mm}, p<0.001)$. In the multivariate analysis, age (standardized beta, 0.342 ; $p<0.001$ ), BMI (standardized beta, 0.083; $p=0.027$ ), presence of hypertension (standardized beta, 0.094; $p=0.013$ ), and presence of low-back pain (standardized beta, 0.104; $p=0.003$ ) were significant determinants of SVA; furthermore, hypertension had an independent effect, result- ing in an increased SVA of $9.5 \mathrm{~mm}$ (Table 5). In other multivariate analysis, only age (standardized beta, 0.128 ; $p=0.005$ ) was a significant determinant of TK; only age (standardized beta, $-0.132 ; p=0.005$ ) was a significant determinant of LL. Age (standardized beta, 0.168; $p<0.001$ ), sex (standardized beta, $0.135 ; p=0.011$ ), height (standardized beta, $-0.308 ; p<0.001$ ), and the presence of low-back pain (standardized beta, $0.108 ; p=0.002$ ) were significant determinants of PT.

\section{Differences in the health-related quality of life be- tween the hypertension and non-hypertension groups}

In all the age subgroups, the EQ-5D was lower in the hypertension group, and the ODI score was higher in the hypertension group; however, these differences were not statistically significant (Tables 2-4). 
Table 4. Difference in spino-pelvic parameter between hypertension and non-hypertension group among 74 to 92 years old population

\begin{tabular}{|c|c|c|c|}
\hline Variable & Hypertension (n=227) & Non-hypertension ( $\mathrm{n}=62$ ) & $p$-value ${ }^{\text {a) }}$ \\
\hline Age (yr) & $80.3 \pm 4.0$ & $79.6 \pm 4.0$ & 0.186 \\
\hline Female percentage (\%) & 59.9 & 48.4 & 0.104 \\
\hline Height (cm) & $150.4 \pm 8.7$ & $153.8 \pm 7.8$ & 0.003 \\
\hline Weight $(\mathrm{kg})$ & $51.5 \pm 8.4$ & $49.0 \pm 8.0$ & 0.034 \\
\hline Body mass index $\left(\mathrm{kg} / \mathrm{m}^{2}\right)$ & $22.7 \pm 2.6$ & $20.7 \pm 2.6$ & $<0.001$ \\
\hline Systolic blood pressure $(\mathrm{mm} \mathrm{Hg})$ & $146.8 \pm 17.6$ & $124.2 \pm 12.3$ & $<0.001$ \\
\hline Diastolic blood pressure $(\mathrm{mm} \mathrm{Hg})$ & $77.5 \pm 11.3$ & $67.1 \pm 10.3$ & $<0.001$ \\
\hline Low-back pain prevalence $(\%)$ & 70.0 & 59.7 & 0.121 \\
\hline Smoking history $(\%)$ & 19.9 & 27.4 & 0.203 \\
\hline \multicolumn{4}{|l|}{ Spino-pelvic parameter } \\
\hline Thoracic kyphosis $\left({ }^{\circ}\right)$ & $37.7 \pm 15.7$ & $38.3 \pm 14.0$ & 0.773 \\
\hline Lumbar lordosis $\left(^{\circ}\right)$ & $37.1 \pm 17.4$ & $38.9 \pm 14.0$ & 0.780 \\
\hline Pelvic tilt $\left(^{\circ}\right)$ & $22.3 \pm 11.5$ & $20.1 \pm 11.2$ & 0.161 \\
\hline Sacral slope $\left({ }^{\circ}\right)$ & $27.4 \pm 11.8$ & $28.0 \pm 9.5$ & 0.764 \\
\hline Pelvic incidence $\left({ }^{\circ}\right)$ & $49.6 \pm 12.2$ & $48.4 \pm 10.7$ & 0.254 \\
\hline Pelvic incidence minus lumbar lordosis $\left({ }^{\circ}\right)$ & $12.5 \pm 20.2$ & $9.5 \pm 14.7$ & 0.307 \\
\hline Sagittal vertical axis (mm) & $70.5 \pm 54.7$ & $57.3 \pm 42.1$ & 0.063 \\
\hline \multicolumn{4}{|l|}{ Health-related quality of life parameter } \\
\hline Euro0ol 5 dimension & $0.78 \pm 0.16$ & $0.81 \pm 0.17$ & 0.124 \\
\hline Oswestry Disability Index & $16.8 \pm 14.0$ & $14.0 \pm 13.0$ & 0.126 \\
\hline
\end{tabular}

Values are presented as mean \pm standard deviation, unless otherwise stated. Values in boldface indicate statistical significance.

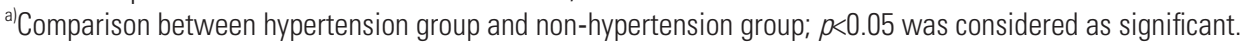

Table 5. Results, multiple regression analysis, predictors of sagittal vertical axis

\begin{tabular}{|c|c|c|c|c|}
\hline \multirow{2}{*}{ Independent variable } & \multicolumn{2}{|c|}{ Unstandardized beta } & \multirow{2}{*}{ Standardized beta } & \multirow{2}{*}{$p$-value } \\
\hline & Coefficient & $95 \%$ Confidence interval & & \\
\hline Age (yr) & 1.982 & 1.497 to 2.466 & 0.342 & $<0.001$ \\
\hline Sex & -5.288 & -15.655 to 5.079 & -0.056 & 0.317 \\
\hline Height $(\mathrm{cm})$ & -0.553 & -1.140 to 0.034 & -0.109 & 0.065 \\
\hline Body mass index $\left(\mathrm{kg} / \mathrm{m}^{2}\right)$ & 1.341 & 0.151 to 2.530 & 0.083 & 0.027 \\
\hline Hypertension & 9.555 & 1.982 to 17.128 & 0.094 & 0.013 \\
\hline Low-back pain & 10.208 & 3.494 to 16.923 & 0.104 & 0.003 \\
\hline Cerebrovascular disease & 1.136 & -13.324 to 15.597 & 0.006 & 0.877 \\
\hline Heart disease & -6.781 & -17.312 to 3.750 & -0.045 & 0.207 \\
\hline
\end{tabular}

Values in boldface indicate statistical significance. A positive value for sex indicates that females were more likely have smaller sagittal vertical axis than males. A positive value for hypertension indicates that people with hypertension were more likely to have higher sagittal vertical axis than people without hypertension. A positive value for low-back pain indicates that people with low-back pain were more likely to have higher sagittal vertical axis than people without low-back pain.

\section{Discussion}

To our knowledge, this is the first study to reveal the asso- ciation between hypertension and forward shift of sagittal plane alignment in middle-aged and elderly individuals. Age is closely related to sagittal plane alignment; therefore, 
we examined it by dividing participants into three age groups to adjust for the influence of age. Thus, we found that hypertension was significantly related to a forward shift in the global sagittal alignment in middle-aged and elderly individuals. It is noteworthy that hypertension was not significantly associated with alignment of the thoracic and lumbar spines. It is reported that the mechanism of spinal sagittal deformity involves the loss of LL due to disc degeneration or osteoporotic vertebral fracture, resulting in a sagittal plane alignment shift forward [7]. The causes of spinal sagittal deformity include disc degeneration [8] and degenerative changes in the muscles [18]; few studies have reported on the relationship of arteriosclerosisrelated diseases, such as hypertension and spinal sagittal deformity. In recent years, obesity has been reported to be associated with a deteriorating sagittal plane alignment $[19,20]$; however, to our knowledge, there is no report on the relationship between hypertension, a very common disease, and sagittal plane alignment. Regarding the association between degenerative musculoskeletal disease and hypertension, Yoshimura et al. [21] have reported on the association between knee osteoarthritis and hypertension in a large cohort study. Moreover, based on a cohort study, Teraguchi et al. [10] revealed that hypertension is associated with disc degeneration. However, hypertension was not related to intervertebral disc degeneration in the cervical and lumbar spines in an age-adjusted multivariate analysis; it was correlated with intervertebral disc degeneration in only the thoracic spine [10]. Hypertension is a factor strongly related to arteriosclerosis [12]. Therefore, it has been suggested that vascular insufficiency of the intervertebral disc due to arteriosclerosis might cause intervertebral disc degeneration [10]. If vascular insufficiency is involved in disc degeneration, disc degeneration will not be limited to one vertebral region. We hypothesized that hypertension produces a sagittal positive shift, resulting in intervertebral disc degeneration. The present results show that hypertension had no statistically significant effect on the local alignment of the thoracic and lumbar spine in middle-aged and elderly people aged $\geq 50$ years; however, it did cause a forward shift of the global sagittal alignment in these subjects. This suggests that hypertension is more closely related to a positive global sagittal alignment than local disc degeneration. After adjusting for age, sex, BMI, and the prevalence of low-back pain, hypertension was an independent factor for an approximately $10-\mathrm{mm}$ forward shift of the SVA. In contrast, hypertension was not a sig- nificant factor for changes in TK, LL, or PT. This supports our hypothesis that postural anomalies occur before disc degeneration in people with hypertension. In general, when the global sagittal alignment is shifted forward, the body attempts to maintain the global sagittal alignment by tilting the pelvis backwards; this is a compensatory mechanism $[22,23]$. However, there was no significant difference in PT between participants with and without hypertension. This indicates that pelvic compensation is more difficult in people with hypertension. The compensation mechanism occurs not only in the pelvis, but also in the lower limbs [22]; therefore, future studies can establish a more detailed mechanism by evaluating the effect of the lower limbs. Hypertension is a leading cause of both, cerebrovascular disease and heart disease [24], and they can be considered confounding factors in this research. Therefore, we performed multivariate analysis to adjust for these factors; however, only hypertension was a significant factor for positive global sagittal alignment.

Regarding the association between hypertension and positive global sagittal alignment, we created the following hypothesis. Hypertension impacts tissue microcirculation, including that in the skeletal muscle [13]. Therefore, the deteriorated microcirculation of the back muscles causes muscle fatigue and muscle degeneration that may result in posture abnormality. These effects may affect the thoracic spine, lumbar spine, and pelvis in small incremental amounts; therefore, the cumulative effect of this may be significantly related to positive global sagittal alignment. Therefore, local alignment like TK or LL may not have statistically different between hypertension group and non-hypertension group. Alternatively, the progression and development of knee osteoarthritis associated with hypertension may be involved in positive global sagittal alignment [21].

Moreover, an increased BMI was an independent risk factor for sagittal anterior shift. This result is consistent with previous reports $[19,20]$. In this study, we also evaluated the health-related QOL. Health-related QOL was worse in people with hypertension than in those without hypertension; however, this difference was not significant.

There are certain limitations of the present study. First, this study was a cross-sectional research. Therefore, it is not possible to clarify the cause in the relationship between hypertension and positive global sagittal alignment in this research. Second, this study involved participants of a musculoskeletal screening project conducted in a 
mountainous area. Therefore, the mechanical stress on the limbs and the spine may differ from those in urban areas. Third, we did not actually evaluate disc degeneration. Therefore, it is difficult to clarify whether positive global sagittal alignment affects disc degeneration or if intervertebral disc degeneration affects positive global sagittal alignment in this study. Fourth, we did not examine the sagittal alignment of the cervicothoracic junction and the lower limbs. We believe that these alignments also affect the global sagittal plane alignment; thus, these aspects require further research. To our knowledge, this is the first study to examine the relationships of atherosclerotic factors and SVA; we plan to perform a longitudinal study to clarify the manner in which hypertension is involved in positive global sagittal alignment.

\section{Conclusions}

To our knowledge, this is the first study to reveal that hypertension is related to forward-shifted global sagittal alignment in middle-aged and elderly people. In the present study, hypertension was not related with alignment of the thoracic and lumbar spines and may be more directly related to global sagittal alignment than local degeneration.

\section{Conflict of Interest}

No potential conflict of interest relevant to this article was reported. DT has a donated fund laboratory by Medtronic Sofamor Danek Inc. (Memphis, TN, USA), Japan Medical Dynamic Marketing Inc. (Tokyo, Japan), and Meitoku Medical Institute Jyuzen Memorial Hospital (Hamamatsu, Japan). SO belongs to a donated fund laboratory by Medtronic Sofamor Danek Inc. (Memphis, TN, USA), Japan Medical Dynamic Marketing Inc. (Tokyo, Japan), and Meitoku Medical Institute Jyuzen Memorial Hospital (Hamamatsu, Japan).

\section{Acknowledgments}

The authors would like to express great thanks to Mr. Katsutoki Obayashi, past Mayer of Toei town, Mr. Koji Murakami, Mayer of Toei town, staff members of Municipality Health and Welfare office. The authors also would like to express great thanks to Ms. Naomi Uchiyama, Ms. Nao Kuwahara, Ms. Kumiko Yano and Mr. Taku Nagao
(Department of Orthopedic Surgery, Hamamatsu University School of Medicine) for their excellent assistance of survey.

\section{References}

1. Glassman SD, Bridwell K, Dimar JR, Horton W, Berven S, Schwab F. The impact of positive sagittal balance in adult spinal deformity. Spine (Phila Pa 1976) 2005;30:2024-9.

2. Schwab F, Dubey A, Pagala M, Gamez L, Farcy JP. Adult scoliosis: a health assessment analysis by SF36. Spine (Phila Pa 1976) 2003;28:602-6.

3. Suzuki N. Studies on postures of healthy Japanese adults: a classification of postures and their relation to change in different age groups. J Jpn Orthop Assoc 1978;52:471-92.

4. Togawa D, Yasuda T, Yamato Y, et al. Toei 2012 study: sagittal spinal alignment and oswestry disability index in volunteers with age over 50. Proceedings of the Annual Meeting of the American Academy of Orthopaedic Surgeons; 2015 Mar 24-28; Las Vegas, USA. Rosemont (IL): American Academy of Orthopaedic Surgeons; 2015.

5. Glassman SD, Berven S, Bridwell K, Horton W, Dimar JR. Correlation of radiographic parameters and clinical symptoms in adult scoliosis. Spine (Phila $\mathrm{Pa}$ 1976) 2005;30:682-8.

6. Bridwell KH, Lewis SJ, Lenke LG, Baldus C, Blanke K. Pedicle subtraction osteotomy for the treatment of fixed sagittal imbalance. J Bone Joint Surg Am 2003;85:454-63.

7. Savage JW, Patel AA. Fixed sagittal plane imbalance. Global Spine J 2014;4:287-96.

8. Habibi Z, Maleki F, Meybodi AT, Mahdavi A, Saberi $\mathrm{H}$. Lumbosacral sagittal alignment in association to intervertebral disc diseases. Asian Spine J 2014;8:8139.

9. Barrey C, Jund J, Noseda O, Roussouly P. Sagittal balance of the pelvis-spine complex and lumbar degenerative diseases: a comparative study about 85 cases. Eur Spine J 2007;16:1459-67.

10. Teraguchi M, Yoshimura N, Hashizume H, et al. Metabolic syndrome components are associated with intervertebral disc degeneration: the Wakayama Spine Study. PLoS One 2016;11:e0147565.

11. Hangai M, Kaneoka K, Kuno S, et al. Factors associ- 
ated with lumbar intervertebral disc degeneration in the elderly. Spine J 2008;8:732-40.

12. Alexander RW. Theodore Cooper Memorial Lecture: hypertension and the pathogenesis of atherosclerosis: oxidative stress and the mediation of arterial inflammatory response: a new perspective. Hypertension 1995;25:155-61.

13. Jung F, Pindur G, Ohlmann P, et al. Microcirculation in hypertensive patients. Biorheology 2013;50:24155.

14. Fairbank JC, Couper J, Davies JB, O'Brien JP. The Oswestry low back pain disability questionnaire. Physiotherapy 1980;66:271-3.

15. EuroQol Group. EuroQol: a new facility for the measurement of health-related quality of life. Health Policy 1990;16:199-208.

16. O’Brien MF, Kuklo TR, Blanke KM, et al. Spinal Deformity Study Group: radiographic measurement manual. Memphis (TN): Medtronic Sofamor Danek USA Inc.; 2005.

17. Banno T, Togawa D, Arima H, et al. The cohort study for the determination of reference values for spinopelvic parameters ( $\mathrm{T} 1$ pelvic angle and global tilt) in elderly volunteers. Eur Spine J 2016;25:3687-93.

18. Moal B, Bronsard N, Raya JG, et al. Volume and fat infiltration of spino-pelvic musculature in adults with spinal deformity. World J Orthop 2015;6:727-37.
19. Jalai CM, Diebo BG, Cruz DL, et al. The impact of obesity on compensatory mechanisms in response to progressive sagittal malalignment. Spine J 2017;17:681-8.

20. Araujo F, Lucas R, Alegrete N, Azevedo A, Barros H. Individual and contextual characteristics as determinants of sagittal standing posture: a population-based study of adults. Spine J 2014;14:2373-83.

21. Yoshimura N, Muraki S, Oka H, et al. Accumulation of metabolic risk factors such as overweight, hypertension, dyslipidaemia, and impaired glucose tolerance raises the risk of occurrence and progression of knee osteoarthritis: a 3-year follow-up of the ROAD study. Osteoarthritis Cartilage 2012;20:1217-26.

22. Barrey C, Roussouly P, Le Huec JC, D’Acunzi G, Perrin G. Compensatory mechanisms contributing to keep the sagittal balance of the spine. Eur Spine J 2013;22:S834-41.

23. Roussouly P, Nnadi C. Sagittal plane deformity: an overview of interpretation and management. Eur Spine J 2010;19:1824-36.

24. Bansal BC, Agarwal AK, Rewari BB. Hypertension and cerebrovascular disease. J Indian Med Assoc 1999;97:226-32. 